\title{
Investigation on Dynamic Characteristic of Geared Rotor System Composed of Ultrahigh Molecular Polyethylene Gear and Metal Gear
}

\author{
Daqian Pang ${ }^{1,2,}$, Changjun $\mathrm{Ma}^{1}$, Xin Zhang ${ }^{1}$, Yue Liu' ${ }^{1}$, Bingxian Zhu ${ }^{1}$ \\ ${ }^{1}$ China North Vehicle Research Institute, Beijing 100072, China \\ ${ }^{2}$ Science and Technology on Vehicle Transmission Laboratory, Beijing 100072, China
}

\begin{abstract}
A geared rotor system model consisting of UPE gears and steel gears for medium speed, medium load and long servo time is proposed. The solid models of steel-steel, steel-UPE and UPE-UPE gear systems were established. The dynamic models of steel-UPE gear system and UPE-UPE gear system were reconstructed by using the theoretical mechanical model of UPE. The time-varying meshing stiffness of three gear systems is analyzed. The vibration responses of three gear systems are studied by STFT method. The dynamic loads of three gear systems under different loads and rotational speeds are researched in detail, and the changing rules of dynamic loads of three gear systems under different loads and rotational speeds are revealed. At last, the first ten modes of three gear systems are calculated and the results are discussed. At the same time, the modal results are used to verify the correctness of the research on vibration response and dynamic load of three gear systems.
\end{abstract}

\section{Introduction}

With the rapid development of logistics industry, the performance requirements of transport equipment are also rising. Low seismic, low noise, high wear-resistant transmission equipment is required by more and more people. For transmission equipment, vibration and noise mostly come from mechanical transmission system, and the main part of mechanical transmission system is gear transmission system. Gear transmission is one of the most important transmissions of the mechanical transmission, and is widely used for its efficiency, structure, and stability. Vibration characteristics of gear-rotor system under multi-body faults are studied by simulation. A majority of the gears used in modern industry is made of metal. Due to the properties of metals, vibration and noise may be relatively obvious during the transmission of gears, which is also one of the causes of faults and invalidation of gears, thus, to reduce vibration and reduce noise of the operating gears has become one of the major researches on improving the performance of the gear transmission system. Active vibration suppression includes enhancing machining accuracy, gear structure and tooth modification, etc., however, these methods cannot eliminate excitation and time-varying stiffness, besides, active vibration suppression has problems including high cost, heavy working load and other problems. While passive vibration suppression applies energy-consuming equipment to absorb energy generated from gear vibration, in order to reduce vibration and reduce noise. Researches on the passive vibration suppression mainly focus on the study of viscoelastic damper and friction damper. Currently, the majority of researches and application of the passive vibration suppression uses particle damping technology; the vibration and noise of the geared rotor system would then be suppressed by adding the particle damping in the cavities of the machinery.

Ultrahigh molecular polyethylene (UPE) is a kind of engineering plastics with linear structure and excellent comprehensive properties, it has the advantages of wear resistance, corrosion resistance, shock resistance, selflubrication, small friction coefficient and low temperature resistance, the average wear rate of different materials is shown in Table 1. Macromolecules are mainly made up of methylene. There are no branched chains and double bonds in the molecular structure, this gives UPE the superior mechanical properties that other conventional molecular weight polyethylene does not have. It is widely used in general transmission equipment, mining and metallurgical equipment, food machinery, medical equipment and artificial joints.

Table 1. Average wear rate of different materials

\begin{tabular}{cc}
\hline Material & Average wear rate(\%) \\
\hline UPE & 0.46 \\
nylon & 1.51 \\
PTEF & 2.31 \\
45\#steel & 4.02 \\
Stainless steel & 4.05 \\
\hline
\end{tabular}

As a new macromolecule polymer material, UPE gear has incomparable advantages under conditions such as

\footnotetext{
$\overline{{ }^{*} \text { Corresponding author: } 328461342 @ q q . c o m}$
} 
medium load, medium speed and low temperature, etc. Most importantly, UPE gear has properties including large damping and small friction coefficient, etc., and its performance in vibration reduction, noise reduction, lightweight and improve power transmission accuracy is more excellent than most metal gears. The output of plastic gears is almost equal to that of metal gears. Previous macromolecule polymer gears rarely used the material of UPE, and the portfolio of metal gear and nonmetal gear is even more rare; this article properly proposes the implementation of a computer stimulation experiment of the three types of the geared rotor system, i.e. steel-steel gear system, steel-UPE gear system and UPE-UPE gear system, under simulated normal operating condition. We will use STFT method to study the dynamic response of the three systems, and make a detailed analysis of the dynamic load of the system under different rotational speeds and loads. Finally, the first ten modes of three gear systems are calculated, and the results of modal calculation are discussed.

\section{Modeling and calculation}

In order to reflect the actual vibration situation, a gear rotor dynamic model is established on the basis of solid geared rotor system. analysis on mechanical characteristics of steel and UPE. The mechanical model of UPE is established.

\subsection{The dynamic model of gear system}

The dynamic model of involute cylindrical gear is generally shown in the Figure 1. According to Newton's mechanics principle, the dynamic equation of involute cylindrical gear is shown in Eq. (1).

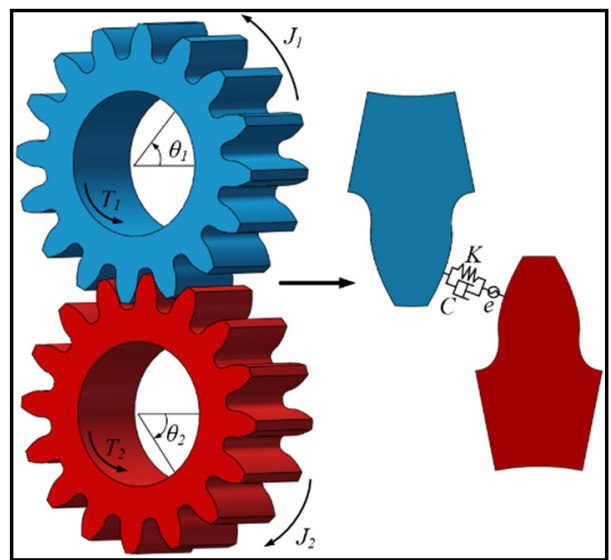

Figure 1. The dynamic model of involute cylindrical gear

$$
\left\{\begin{array}{l}
J_{1} \ddot{\theta}_{1}=T_{1}-K r_{b 1}\left(r_{b 1} \theta_{1}-r_{b 2} \theta_{2}-e\right)-C r_{b 1}\left(r_{b 1} \dot{\theta}_{1}-r_{b 1} \dot{\theta}_{2}-\dot{e}\right) \\
J_{2} \ddot{\theta}_{2}=-T_{2}+K r_{b 2}\left(r_{b 1} \theta_{1}-r_{b 2} \theta_{2}-e\right)+C r_{b 2}\left(r_{b 1} \dot{\theta}_{1}-r_{b 1} \dot{\theta}_{2}-\dot{e}\right)
\end{array}\right.
$$

Where, $\mathrm{r}_{\mathrm{b} 1}$ is the radius of base circle of driving gear; $\mathrm{rb} 2$ is the radius of base circle of driven gear; $J_{\mathrm{i}}$ is the moment of inertia; $\theta_{\mathrm{i}}$ is the rotation angle of the gears; Subscript 1 and 2 denotes driving and driven gear; $T_{1}$ is the torque applied to the driving gear; $T_{2}$ is the torque applied to the driven gear.

\subsection{The curvature radius of gear pair}

The dimensionless coordinate $\Gamma$ is introduced, which can represent any position on the meshing line. The curvature radius of any position is deduced with dimensionless coordinate as variable, so that the accurate contact stress can be obtained, as shown in Figure 2. Taking theoretical meshing line $\mathrm{N}_{1} \mathrm{~N}_{2}$ as reference axis, node $\mathrm{C}$ as coordinate origin, $\mathrm{CN}_{2}$ direction as coordinate positive direction, and dimensionless coordinate of any node $\mathrm{Y}$ on meshing line as follows:

$$
\Gamma_{Y}=\frac{\overline{C Y}}{N_{1} C}=\frac{\overline{N_{1} Y}-\overline{N_{1} C}}{\overline{N_{1} C}}=\frac{r_{b 1} \tan a_{y 1}-r_{b 1} \tan a}{r_{b 1} \tan a}=\frac{\tan a_{y 1}}{\tan a}-1
$$

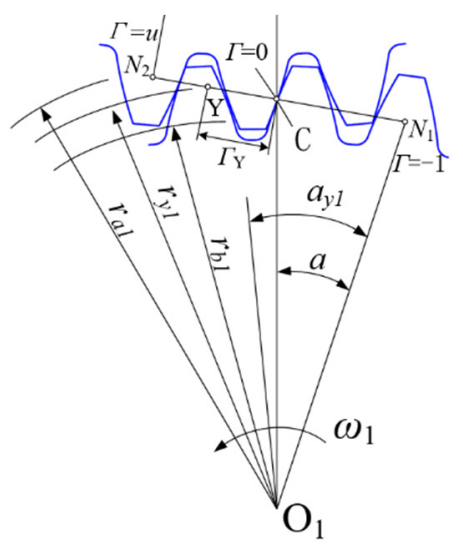

Figure 2. The dimensionless coordinate of gear.

$$
a_{y 1}=\arccos \left(\frac{r_{b 1}}{r_{y 1}}\right)
$$

Where, $\mathrm{r}_{\mathrm{b} 1}$ is radius of base circle of driving gear, $\mathrm{r}_{\mathrm{y} 1}$ represents the pitch radius of gear, $a$ is the pressure angle of pitch circle, $a_{\mathrm{y} 1}$ represents the pressure angle at any point of the driving gear.

The comprehensive curvature radius of gear $\rho_{\text {red }}$

$$
\frac{1}{\rho_{\text {red }}}=\frac{(u+1) \frac{a_{y 1}}{u+1} \cdot \frac{\sin a}{\cos \beta}}{(1+\Gamma)(u-\Gamma)\left(\frac{a_{y 1}}{u+1} \cdot \frac{\sin a}{\cos \beta}\right)^{2}}=\frac{(u+1)^{2}}{(1+\Gamma)(u-\Gamma) a_{y 1}} \cdot \frac{\cos \beta}{\sin a}
$$

In the process of meshing between the driving and the driven gears, the tangential velocity of the contact line is inconsistent, which results in the relative sliding speed. The existence of relative sliding velocity leads to the generation of friction heat flux on the tooth surface.

$v_{1}$ and $v_{2}$ are the absolute velocity of the driving and driven gears along the tangent direction of the meshing surface at the any meshing point

$$
\begin{aligned}
& v_{1}=\rho_{1} \omega_{1}=\frac{\pi n_{1}}{30}(1+\Gamma) \frac{d_{1}}{2} \sin a=v^{\prime}(1+\Gamma) \sin a \\
& v_{2}=\rho_{2} \omega_{2}=\frac{\pi n_{2}}{30}(u-\Gamma) \frac{d_{2}}{2} \sin a=v^{\prime}\left(1-\frac{\Gamma}{u}\right) \sin a
\end{aligned}
$$

Where, $\omega_{1}$ and $\omega_{2}$ are the angular velocity of the driving and the driven gears $(\mathrm{r} / \mathrm{s}) ; n_{1}$ is the speed of the driving gear( $\mathrm{rad} / \mathrm{s}) ; v^{\prime}$ denotes the pitch speed; $\Gamma$ is the dimensionless coordinates of any meshing position of gear pair. 


\subsection{Calculation of contact stress}

According to the Hertz contact stress formula, the parallel cylinder contact stress model is shown in Figure 3. Contact half width $b_{H}$ in Fig 3 is

$$
\begin{gathered}
\mathrm{b}_{H}=\sqrt{\frac{4 F}{\pi L} \times \frac{\frac{1-v_{1}^{2}}{E_{1}}+\frac{1-v_{2}^{2}}{E_{2}}}{\frac{1}{\rho_{\text {red }}}}} \\
\rho_{\text {red }}=\left(\frac{1}{\rho_{1}}+\frac{1}{\rho_{2}}\right)^{-1}
\end{gathered}
$$

where, $F$ is the normal pressure; $L$ is the length of the contact line; $v_{1}$ and $v_{2}$ are Poisson's ratio of two cylinder materials, while $E_{1}$ and $E_{2}$ are the modulus of elasticity concerning two cylindrical materials.

Using dimensionless parameter $\Gamma$ to represent contact half width:

$$
\begin{aligned}
& \mathrm{b}_{H}=\sqrt{\frac{4 F}{\pi L} \times \frac{\frac{1-v_{1}^{2}}{E_{T, 1}}+\frac{1-v_{2}^{2}}{E_{T, 2}}}{\frac{1}{\rho_{\text {red }}}}}=\sqrt{\frac{8 F \rho_{\text {red }}}{\pi L E_{r e d}}} \\
& \frac{1}{E_{\text {red }}}=\frac{1}{2}\left(\frac{1-v_{1}^{2}}{E_{T, 1}}+\frac{1-v_{2}^{2}}{E_{T, 2}}\right)
\end{aligned}
$$

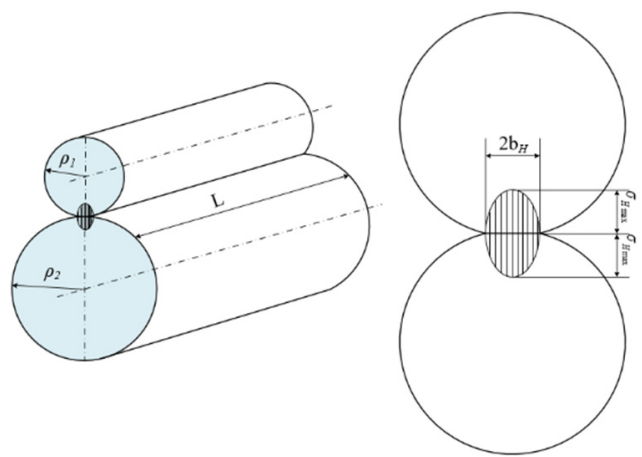

Figure 3. Contact Stress of the Parallel Cylinder

The resultant force of stress on the contact surface should be balanced with external force $\mathrm{F}$, so

$$
\begin{gathered}
F=\frac{\pi \sigma_{H \max } \mathrm{b}_{H} L}{2} \\
\sigma_{H \max }=\frac{2 F}{\pi \mathrm{b}_{H} L}
\end{gathered}
$$

Bringing Eq. (4), (9) into Eq. (12), the basic equation of basic stress can be obtained as

$$
\sigma_{H \max }=\sqrt{\frac{8 F \sin a(1+\Gamma)(u-\Gamma) a_{y 1}}{\pi L \cos \beta(u+1) \cdot E_{r e d}}}
$$

\subsection{The dynamic model of UPE}

Because the UPE is a solid linear polymer. Therefore, the viscoelastic model usually as the three element model is shown in Figure 4(a). The mechanical model of any viscoelastic body can be considered as a combination of the most basic Maxwell model and the Kelvin model as shown in Figure 4(b) and Figure 4(c) (a)

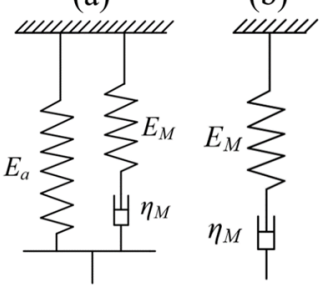

(c)

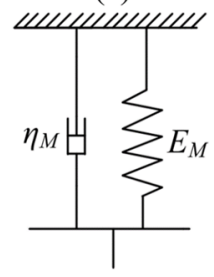

(d)

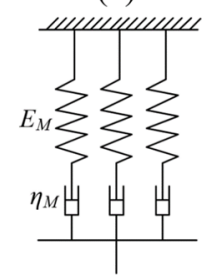

Figure 4. The dynamic model of UPE: (a) Standard linear viscoelastic solids three element model, (b) Maxwell mechanical model, (c) Kelvin mechanical model, (d) Multiple Maxwell two element parallel model

The model is usually referred as a standard linear solid viscoelastic model by Zener. During the creep, the two springs stretch, therefore

$$
\tau^{\prime}=\eta_{M}\left(\frac{1}{E_{\mathrm{a}}}+\frac{1}{E_{M}}\right)
$$

In the stress relaxation process, the dashpot $\eta_{M}$ is still working, so

$$
\tau=\frac{\eta_{M}}{E_{M}}
$$

The stress strain differential equation of the three element model is

$$
\sigma+\tau \frac{\mathrm{d} \sigma}{\mathrm{d} t}=E_{\mathrm{a}} e+\left(E_{\mathrm{a}}+E_{M}\right) \tau \frac{\mathrm{d} e}{\mathrm{~d} t}
$$

According to the principle of Boltzmann superposition, the equation of stress relaxation is

$$
\sigma(t)=\left[G_{r} e\right]+\int_{-\infty}^{t} G(t-\tau) \frac{\mathrm{de}(\tau)}{\mathrm{d} \tau} \mathrm{d} t
$$

In the Eq. (17), $G_{r}$ is Equilibrium modulus

Although the dynamic of viscoelastic solids described by the three element model is more practical than that of the two element, it can only give an exponential response with a relaxation time. In order to make the relaxation behavior of viscoelastic solids described model more similar to the polymer viscoelastic body. The Maxwell parallel complex models are presented, as shown in Figure 4(d).

For stress relaxation under constant strain conditions, the Maxwell model is

$$
\sigma(t)=e E \exp \left(-\frac{t}{\tau}\right)
$$

Thus, the stress relaxation modulus is

$$
G(t)=E \exp \left(-\frac{t}{\tau}\right)
$$

If the element strain of Maxwell model is e, for model

$$
\sigma(t)=e \sum^{n} E_{n} \exp \left(-\frac{t}{\tau_{n}}\right)
$$

Use integral instead of summation

$$
\sigma(t)=\left[G_{r} e\right]+e \int_{0}^{\infty} f(\tau) \exp \left(-\frac{t}{\tau}\right) \mathrm{d} \tau
$$

In the Eq. (21), The first term is defined as the final relaxation stress, the spring constant $E_{n}$ is replaced by the weight function $f(\tau) d \tau$, it determines the relaxation time for $\tau$ to $\tau+d \tau$ of Maxwell model element Fraction 
The stress relaxation modulus is defined as

$$
G(t)=G_{r}+\int_{0}^{\infty} f(\tau) \exp \left(-\frac{t}{\tau}\right) \mathrm{d} \tau
$$

According to multiple Maxwell two element parallel model, the dynamic models of steel-UPE system and UPE-UPE system are shown in Figure 5.

(a)

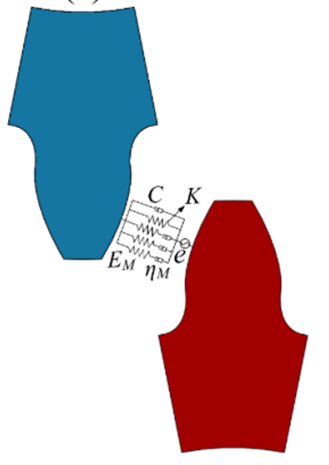

Figure 5. The dynamic model of gear systems: (a) steel-UPE gear system, (b) UPE-UPE gear system

\subsection{The dynamic model of UPE}

The time-varying meshing stiffness of steel gear system has been studied by many people, but the time-varying meshing stiffness of steel-UPE system and UPE-UPE system has rarely been reported. Time-varying meshing stiffness is the main parameter of the dynamic characteristics of gear system. Analyzing the timevarying meshing stiffness of the system can better understand and study the dynamic characteristics of the system. Figure 6 shows the time-varying meshing stiffness of three gear systems. It can be seen that the steel-steel system has the largest time-varying meshing stiffness, while the steel-UPE system and the UPE-UPE system are relatively small. This shows that when gears mesh, the vibration response and dynamic load fluctuation of steel-UPE system and UPE-UPE system are smaller than that of steel-steel system.

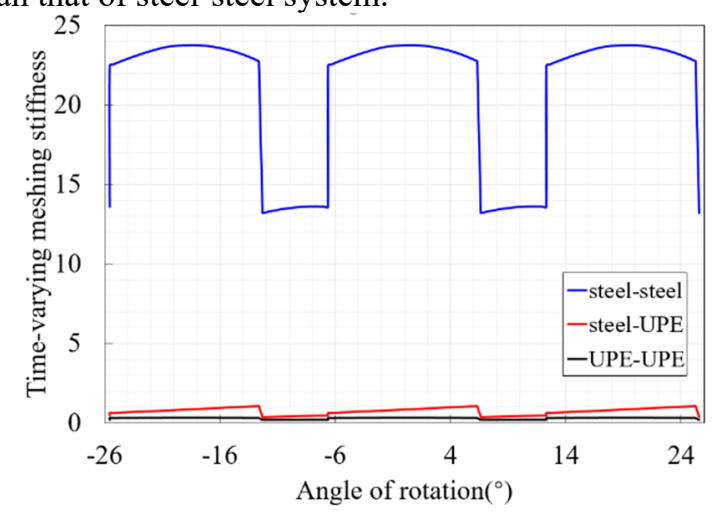

Figure 6. Time-varying meshing stiffness of three gear systems

\section{Modeling and solving process of geared rotor system}

In order to make the simulation data closer to the actual situation, we choose the geared rotor system used in the reducer of the existing logistics transmission equipment to carry out entity modeling and simulation analysis. Only by analyzing the actual system can we get the most accurate experimental data.

\subsection{Modeling and parameter design of geared rotor system}

We select the geared rotor system in the gear reducer as the modeling object. The dimensioning schematic model of gear-rotor system is shown in Figure 7, and the gear parameters are shown in Table 2. According to the size of Figure 7, three different three-dimensional models of gear-rotor system are established, including steel-steel gear system, steel-UPE gear system and UPE-UPE gear system. Steel-Steel represents that both the driving gear and the driven gear are made of steel. Steel-UPE represents that the driving gear is made of steel and the driven gear is made of UPE. UPE-UPE represents that both the driving gear and the driven gear are made of UPE. $3 \mathrm{D}$ models of three geared rotor system are shown in Table 3 .

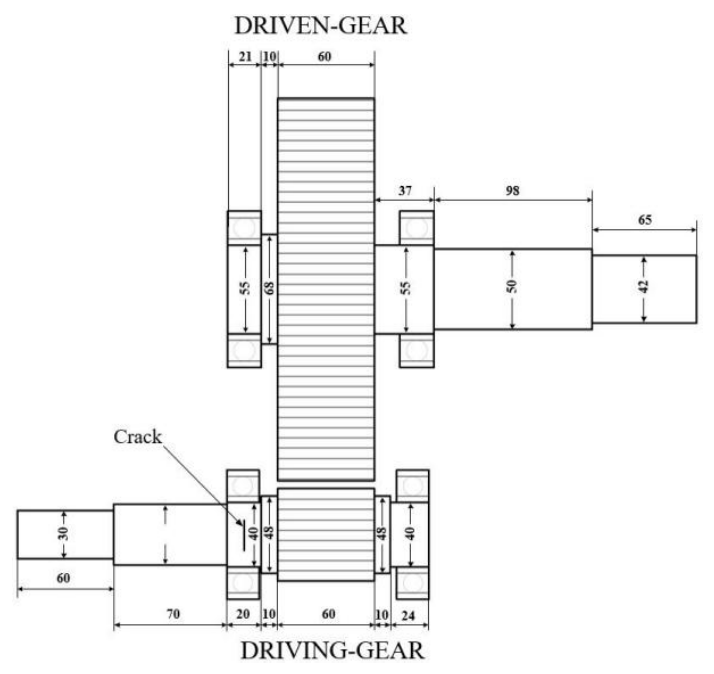

Figure 7. 2D drawing of the geared rotor system

Table 2. Parameter of the geared rotor system

\begin{tabular}{llll}
\hline parameters & $\begin{array}{l}\text { Driving } \\
\text { gear }\end{array}$ & $\begin{array}{l}\text { Driven } \\
\text { gear }\end{array}$ \\
\hline Number of teeth & 19 & 79 \\
Module(mm) & 3 & 3 \\
Pressure angle(deg.) & & 20 & 20 \\
$\begin{array}{l}\text { Young'smodulus, } \\
\left.\text { E(N/mm }{ }^{2}\right)\end{array}$ & Steel & $2.07 \times 10^{5}$ & $2.07 \times 10^{5}$ \\
& UPE & $1.9 \times 10^{4}$ & $1.9 \times 10^{4}$ \\
Poisson's ratio & Steel & 0.29 & 0.29 \\
& UPE & 0.44 & 0.44 \\
\hline
\end{tabular}


Table 3. 3D Model of Geared rotor system

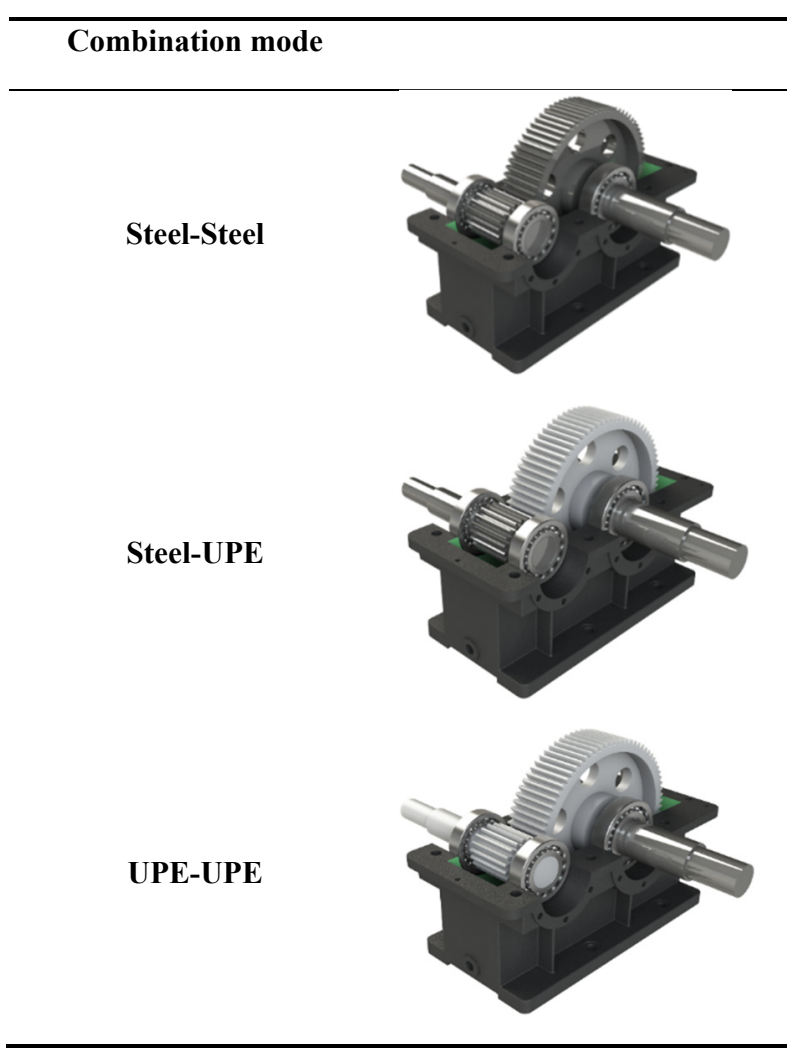

\subsection{Discussion on contact problems}

In the process of simulation, the setting of contact force is very important. It is possible to obtain the simulation results consistent with the fact when the contact force of the gear is calculated correctly. There are two ways to set contact force, including IMPACT function and RESTITUTION function. According to the object of this study, we choose IMPACT function as the contact force calculation method in this paper.

The function expression of the IMPACT is as follows $F= \begin{cases}\operatorname{MAX}\left\{0, k\left(q_{0}-q\right)^{e}-c_{\max } \times \frac{d q}{d t} \times \operatorname{STEP}\left(q, q_{0}-\delta_{\max }, 1, q_{0}, 0\right)\right\} & q<q_{0} \\ 0 & q>q_{0}\end{cases}$

where $q$ is the actual distance between the two contact objects; $\frac{d q}{d t}$ is the change rate over time of the actual distance between two contact object; $q_{0}$ is the reference distance of the two contact objects; based on the abovementioned expression: when $q>q_{0}$, the two objects do not strike, the striking force would be zero; when $q<q_{0}$, the two objects strike, and the striking force of the contact is jointly determined by stiffness $K$, penetration $q_{0^{-}} q$, striking force index $e$, maximum damping $c_{\max }$ and penetration depth $\delta_{\max }$.

According to the Hertz elastic contact theory

$$
\delta=\frac{a^{2}}{R}=\left(\frac{9 P^{2}}{16 R E^{* 2}}\right)^{\frac{1}{3}}
$$

the relationship of the normal force $P$ and transformed $\delta$ is:

$$
P=K \delta^{\frac{3}{2}}
$$

where $K$ is the contact stiffness, which is subject to the material, structure and shape of the contact objects.

$$
\begin{aligned}
& K=\frac{4}{3} R^{\frac{1}{2}} E^{*} \\
& \frac{1}{R}=\frac{1}{R_{1}}+\frac{1}{R_{2}}
\end{aligned}
$$

Where $R_{1}, R_{2}$ are the contact radius of the contact point between the two contact objects.

$$
\frac{1}{E^{*}}=\frac{\left(1-\mu_{1}^{2}\right)}{E_{1}}+\frac{\left(1-\mu_{2}^{2}\right)}{E_{2}}
$$

Where $E_{1}, E_{2}$ are the modulus of the two contact objects, $\mu_{1}, \mu_{2}$ stand for the Poisson ratio of the two contact objects.

\section{Calculating Examples and Result Analysis}

In this section, three different combinations of gear systems, i.e. steel-steel, steel-UPE and UPE-UPE are simulated and analyzed. The time domain, frequency domain and dynamic loads of the gear system under different operating conditions were analyzed. In order to reduce the interference of vibration to the system and obtain more accurate frequency response, the vibration of bearing in working state is neglected in this paper. The simulation model is shown in Figure 8.

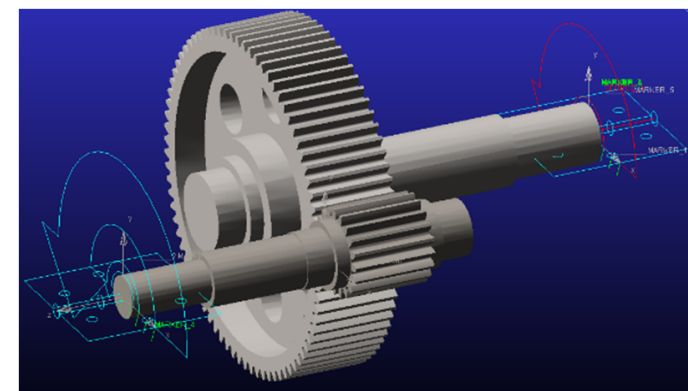

Figure 8. Simulating geared rotor system

\subsection{STFT spectra analysis of geared rotor system under three combinations}

A 600rpm speed is applied to the driving gear and a 500 $\mathrm{Nm}$ load is loaded on the driven gear. The vibration characteristics of three combined modes under the same working conditions are analyzed. For the traditional Fourier transform, only the frequency and amplitude of the system can be seen, but the frequency spectrum along the time direction is not displayed. Therefore, STFT method is used to study the vibration characteristics of the system. STFT can not only see the frequency and corresponding amplitude, but also show the change of frequency points along the time direction, so as to analyze the vibration response of the system more accurately. 


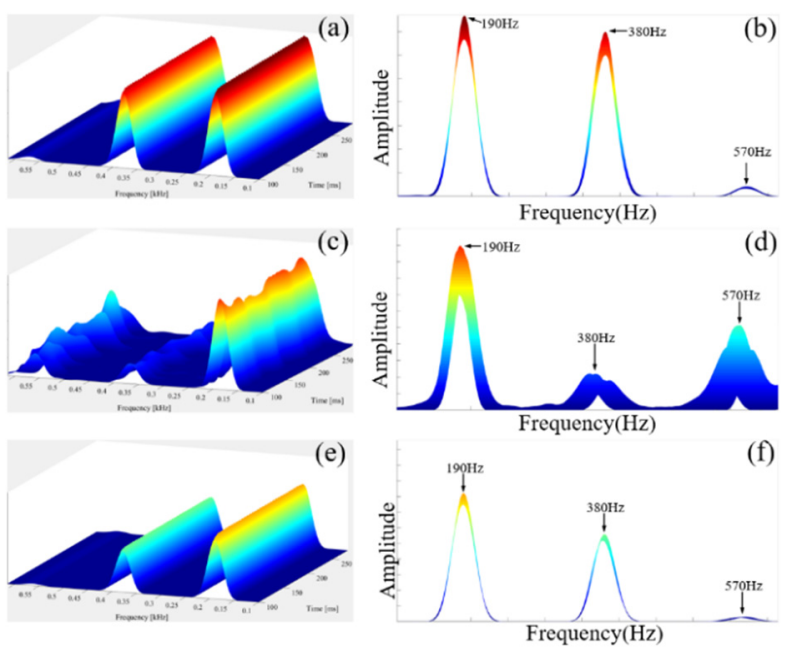

Figure 9. STFT spectra of Geared rotor system

The STFT spectra of three different combinations of geared rotor systems are shown in Figure 10. Figure 9(a)(b) represents the STFT spectra of steel-steel geared rotor system, Figure 9(c)(d) represents the STFT spectra of steel-UPE geared rotor system, Figure 9(e)(f) represents the STFT spectra of steel-UPE geared rotor system. As can be seen from Figure 9, under the same rotational speed and load, the vibration of UPE-UPE system is the smallest, while that of steel-steel system is the largest. The energy of main frequency and double frequency of steel-steel and UPE-UPE systems is basically unchanged along the time direction, while the position of main frequency and double frequency of steelUPE system fluctuates obviously along the time direction. This is because the elastic modulus and Poisson's ratio of the two materials are quite different, so the position of the main frequency and double frequency will fluctuate along the time direction, but this will not increase the vibration of the whole system, because the main frequency and double frequency have a significant decrease compared with steel-steel system.

\subsection{Dynamic load analysis of geared rotor system under three combinations}

For the vibration characteristics of geared rotor system, it is very necessary to analyze the dynamic load. From the dynamic load spectrum, we can see the load changes and the response changes in the meshing process very clearly. In this section, the dynamic loads of three systems are studied, which are loaded with different rotational speeds and loads. The dynamic loads of the three systems are analyzed with the increase of rotational speed and load. We applied 600rpm, 1200rpm and 1800rpm rotational speeds to the driving wheel and 300, 500 and $700 \mathrm{Nm}$ loads to the driven gear respectively.

\subsubsection{Dynamic Load Characteristic of Three Systems at Different Rotational Speed}

It can be seen from Figure 10 that the dynamic load amplitude of steel-UPE system is very small under 300 $\mathrm{Nm}$ load. This is because the damping of UPE is greater than that of steel and its stiffness is smaller. On the other wise, UPE has the ability of self-lubrication, which can effectively reduce the change of meshing stiffness when the gear meshes. As can be seen from Figure 10, the dynamic load amplitude of UPE-UPE system is very close to that of steel-UPE system. It shows that when a pair of gears engage, as long as one of them is UPE material, the dynamic load amplitude of the system can be reduced.
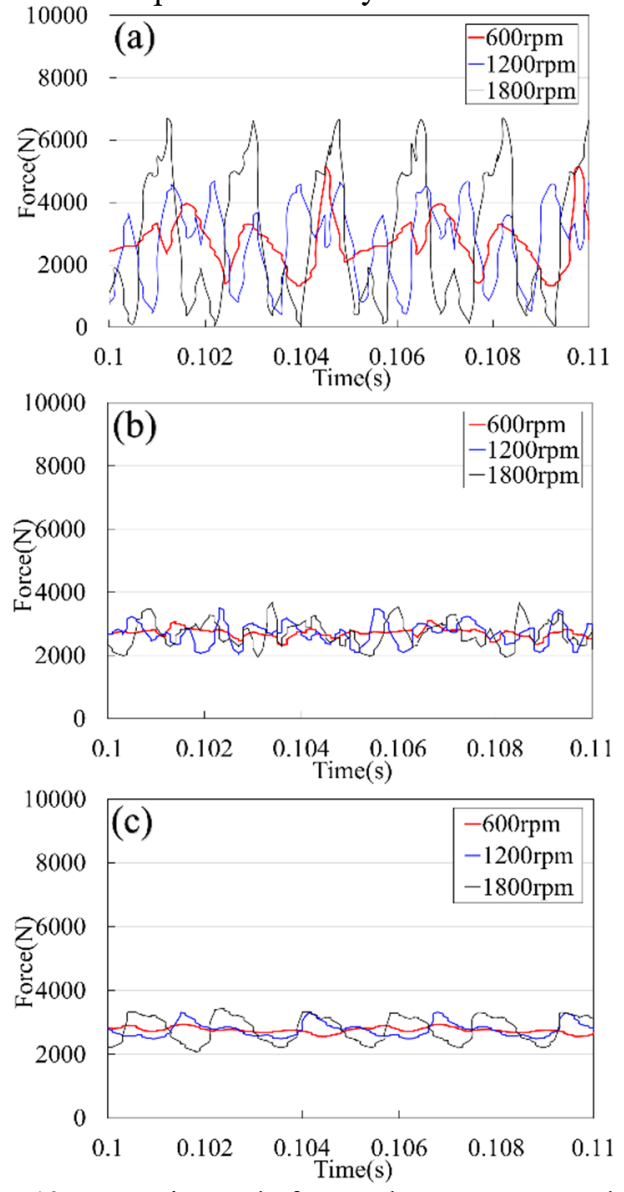

Figure 10. Dynamic Load of Geared rotor system under 300 Nm Load: (a) steel-steel, (b)steel-UPE, (c) UPE-UPE

It is observed that the dynamic load amplitude of steelsteel system at $600 \mathrm{rpm}$ and $1200 \mathrm{rpm}$ differ slightly. The phenomenon of steel-UPE system is just reverses, the dynamic load amplitudes of $600 \mathrm{rpm}$ and $1200 \mathrm{rpm}$ differ greatly, while those of $1200 \mathrm{rpm}$ and $1800 \mathrm{rpm}$ differ slightly. The same rule also appears in UPE-UPE system. It shows that the dynamic load sensitivity of steel-steel system is not good at low speed. Only when the system reaches a relatively high speed, the dynamic load of steelsteel system will change greatly. The sensitivity of steelUPE system and UPE-UPE system is relatively higher at low speed.

Figure 11 shows the dynamic load spectrum of three systems at different rotational speeds under $500 \mathrm{Nm}$ load. It can be seen that when the load changes, the dynamic load amplitude of the three systems does not change. The steel-steel system has the largest amplitude and the UPEUPE system has the smallest amplitude. The difference is that when the load changes to $500 \mathrm{Nm}$, the sensitivity of the dynamic load of different systems to the speed has changed. When the load is $300 \mathrm{Nm}$, the dynamic load amplitude of steel-UPE system varies greatly between 
$600 \mathrm{rpm}$ and $1200 \mathrm{rpm}$. But when the load is $500 \mathrm{Nm}$, the difference of dynamic load amplitude between $600 \mathrm{rpm}$ and $1200 \mathrm{rpm}$ of steel-UPE system is much smaller. The same phenomenon occurs in UPE-UPE systems.
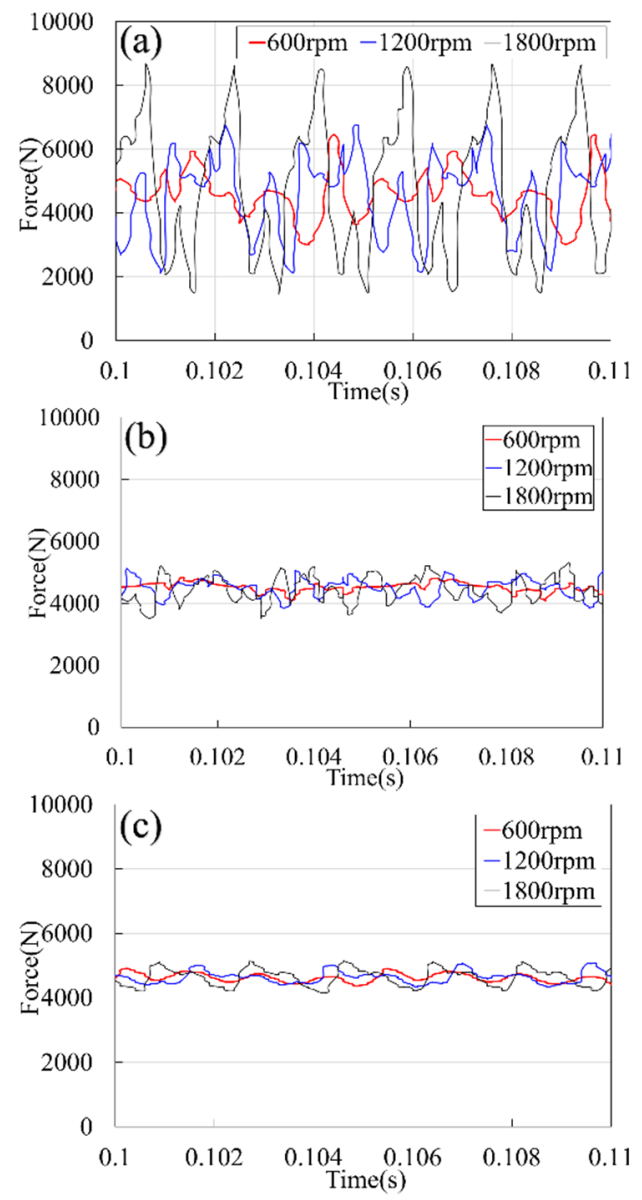

Figure 11. Dynamic Load of Geared rotor system under 500 Nm Load: (a) steel-steel, (b)steel-UPE, (c) UPE-UPE

Figure 12 shows the dynamic load spectrum of three systems at different rotational speeds under $700 \mathrm{Nm}$ load. The dynamic load amplitude of steel-UPE system at 600 rpm and $1200 \mathrm{rpm}$ are not very different under $700 \mathrm{Nm}$ load. But the dynamic load amplitude of $1200 \mathrm{rpm}$ and $1800 \mathrm{rpm}$ is much larger than that of $500 \mathrm{Nm}$. In addition, we also find an interesting phenomenon that the dynamic load amplitude of UPE-UPE system is basically the same at three rotational speeds under $700 \mathrm{Nm}$ load.
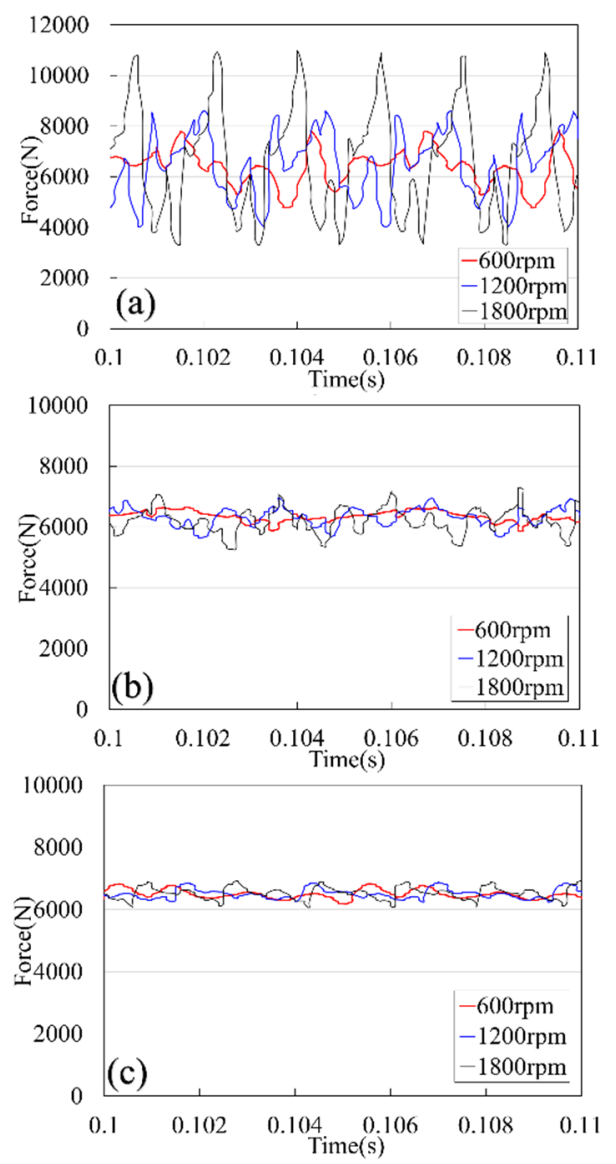

Figure 12. Dynamic Load of Geared rotor system under 700 Nm Load: (a) steel-steel, (b)steel-UPE, (c) UPE-UPE

In order to more clearly see the dynamic load changes of the three systems under different rotational speeds, we extract the fluctuation of the dynamic load amplitude, as shown in Figure 13. It can be seen from the figure that the dynamic load fluctuation of steel-steel system will increase obviously at $1200 \mathrm{rpm}$ under $300 \mathrm{Nm}$ load. Although the dynamic load fluctuation of steel-steel system at $500 \mathrm{Nm}$ is greater than that of $700 \mathrm{Nm}$ under $1200 \mathrm{rpm}$, when the speed reaches $1800 \mathrm{rpm}$, the fluctuation is less than that of $700 \mathrm{Nm}$. The dynamic load fluctuation of steel-UPE system and UPE-UPE system increases linearly with the increase of rotational speed at $300 \mathrm{Nm}$. When the load is $500 \mathrm{Nm}$, the increase rate of dynamic load fluctuation of steel-UPE system is higher than that of $300 \mathrm{Nm}$. The dynamic load fluctuation of UPE-UPE system is relatively low. The dynamic load fluctuation law of steel-UPE system at $700 \mathrm{Nm}$ is similar to that at $500 \mathrm{Nm}$. However, the dynamic load fluctuation of UPE-UPE system is basically unchanged, and it also decreases at $1200 \mathrm{rpm}$. 


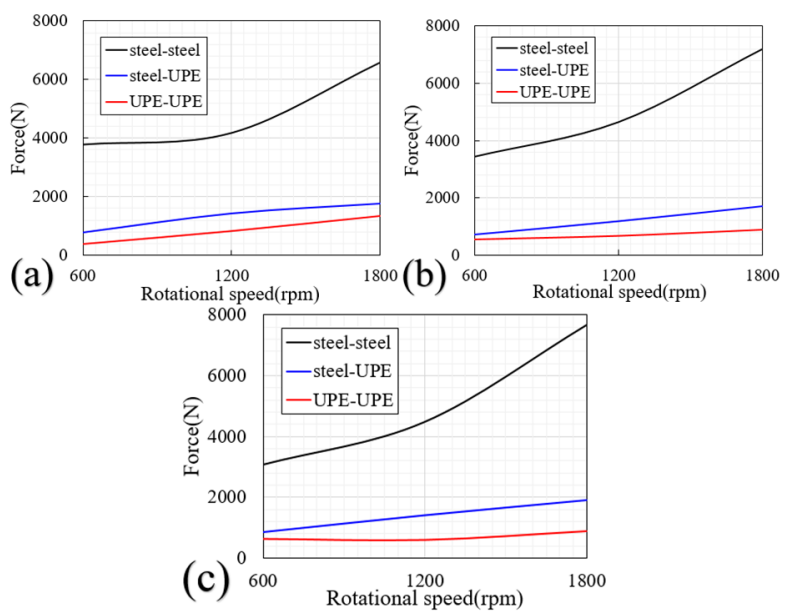

Figure 13. Dynamic load fluctuation of three systems at different rotational speed under different loads: (a) $300 \mathrm{Nm}$, (b) $500 \mathrm{Nm}$, (c) $700 \mathrm{Nm}$

\subsubsection{Dynamic load characteristic of three systems at different load}

It can be seen from Figure 14(a) that the dynamic load fluctuation of steel-steel system decreases gradually with the increase of load at 600rpm. The dynamic load fluctuation of steel-UPE system decreases only at $500 \mathrm{Nm}$, and remains unchanged at $300 \mathrm{Nm}$ and $700 \mathrm{Nm}$. The dynamic load fluctuation of UPE-UPE system increases slowly with the increase of load at 600rpm.

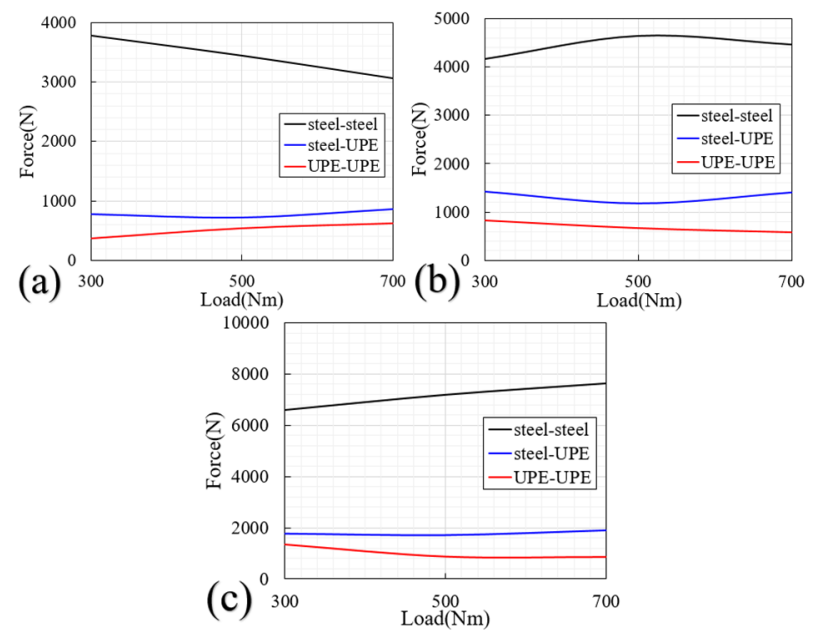

Figure 14. Dynamic load fluctuation of three systems at different load under different rotational speeds: (a) 600rpm, (b) 1200rpm, (c) $1800 \mathrm{rpm}$

According to Figure 14(b), the dynamic load fluctuation of steel-steel system increases first and then decreases with the increase of load at $1200 \mathrm{rpm}$. On the contrary, the dynamic load fluctuation of steel-UPE system decreases first and then increases. The dynamic load fluctuation of UPE-UPE system decreases with the increase of load. From Figure 14(c), we can see that the load fluctuation of steel-steel system increases slowly with the increase of load when the speed is $1800 \mathrm{rpm}$. Dynamic load fluctuation of steel-UPE system decreases first and then increases. The dynamic load fluctuation of UPE-UPE system decreases gradually when the load is less than $500 \mathrm{Nm}$, and remains unchanged when the load is more than $500 \mathrm{Nm}$.

\subsection{Modal analysis}

As an important dynamic characteristic analysis method, modal analysis has been widely used in various fields. In order to verify the vibration characteristics of the three systems, the modal calculation results of the three gear systems are discussed in this section. Table 4 shows the 10th order modal shape of three gear systems.

Table 4. Modal shape of three systems

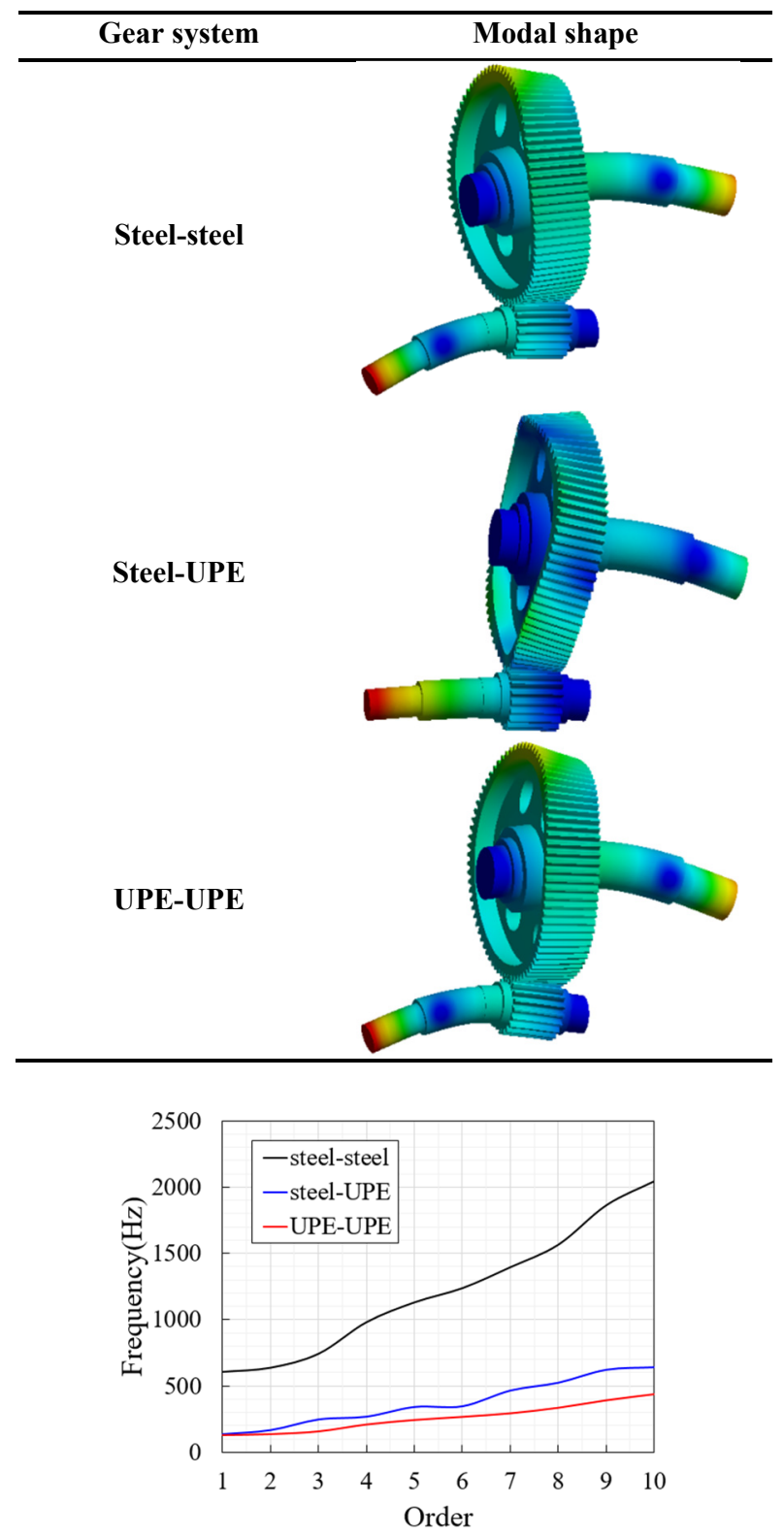

Figure 15. The first ten natural frequencies of three systems

It can be seen that the natural frequencies of the three systems increase gradually, as shown in Figure 15. The natural frequency of Steel-steel system is highest and UPE-UPE system has the lowest natural frequency. The trend of modal calculation results is the same as that of vibration and dynamic load analysis before. It can be seen 
that although the natural frequency of steel-UPE system is gradually increasing, the fluctuation is larger than that of the other two systems.

\section{5 conclusion}

In this paper, the dynamic characteristics of geared rotor system composed of ultra-high molecular polyethylene gear and metal gear are analyzed. The vibration response of the system is analyzed by STFT method under constant load and rotational speed. The effects of different rotational speeds and loads on the three systems are also studied. Finally, the modal calculation results of the three systems are discussed, and the natural frequencies of the three systems at different orders are revealed.

(1) A gear-rotor system with polyethylene gears and steel gears for medium speed, medium load and high servo time is proposed, and the theoretical dynamic model calculation method of polyethylene is given. Three solid 3D models of gear systems are established. The timevarying meshing stiffness of three systems is calculated.

(2) The dynamic response of three systems under constant speed and load is analyzed by STFT method. It can be found from STFT spectra that steel-UPE system and UPE-UPE system can effectively reduce the vibration of gear system without other disturbances.

(3) The dynamic loads of three gear systems under different rotational speeds and loads are analyzed. We find that the dynamic load fluctuation of the three systems increases monotonously under different loads, but the increase rate varies at different rotational speeds. Under different rotational speeds, the fluctuations of dynamic loads of the three systems tend to be non-linear, which may increase or decrease under different loads.

(4) The modal calculation results of three gear systems are discussed. The natural frequencies of the system at different orders are revealed, and the previous studies on vibration response and dynamic load are fully verified by modal analysis.

\section{References}

1. Amarnath M, Lee Sang-Kwon. Measurement 76, 12 (2015)

2. Chen SY, Tang JY, Wu LJ. Mechanism and Machine Theory 74, 4 (2014)

3. Chey MH, Chase JG, Mander JB, et al..Part II, energy-dissipation and damage reduction. Frontiers of Structural and Civil Engineering 9, 3 (2015)

4. Debnath S, Ranade R, Wunder SL, et al. Journal of applied polymer science 96, 5 (2005)

5. Duan Y, Chen Q. Journal of Vibration Engineering 24, 2 (2011)

6. Eritenel T, Parker RG. Journal of Sound Vibration 331, 15 (2012)

7. Evans SM, Keogh PS. Tribology International 97, 5 (2016)

8. Friend RD, Kinra VK. Journal of Sound and Vibration 233, 1 (2000)
9. Gao P, Mackley MR . Polymer 35, 24 (1994)

10. Kurushin MI, Balyakina VB, Kurushin SA. Procedia Engineering 106 (2015)

11. Li W, Pang D . Journal of Vibration and Control 25, 2 (2019)

12. Liang X, Zuo MJ, Pandey M. Mechanism and Machine Theory 76, 6 (2014)

13. Liu G, Parker RG. Journal of Sound and Vibration 320, 4-5 (2009)

14. Lu Z, Lu XL, Masri SF. Journal of Sound and Vibration 329, 26 (2010) 